\title{
Photoresponsive DNA nanocapsule having an open/close system for capture and release of nanomaterials.
}

\section{AUTHOR(S):}

Takenaka, Tomohiro; Endo, Masayuki; Suzuki, Yuki; Yang, Yangyang; Emura, Tomoko; Hidaka, Kumi; Kato, Takayuki; Miyata, Tomoko; Namba, Keiichi; Sugiyama, Hiroshi

\section{CITATION:}

Takenaka, Tomohiro ...[et al]. Photoresponsive DNA nanocapsule having an open/close system for capture and release of nanomaterials.. Chemistry - A European Journal 2014, 20(46): 14951-14954

\section{ISSUE DATE:}

2014-09-15

URL:

http://hdl.handle.net/2433/198829

\section{RIGHT:}

This is the peer reviewed version of the following article: Takenaka, T., Endo, M., Suzuki, Y., Yang, Y., Emura, T., Hidaka, K., Kato, T., Miyata, T., Namba, K. and Sugiyama, H. (2014), Photoresponsive DNA Nanocapsule Having an Open/Close System for Capture and Release of Nanomaterials. Chem. Eur. J., 20: 14951-14954, which has been published in final form at http://dx.doi.org/10.1002/chem.201404757. This article may be used for non-commercial purposes in accordance with Wiley Terms and Conditions for Self-Archiving.; 許諾条件により本文ファイルは2015-09-15に公開.; の論文は出版社版でありません。引用の際には出版社版をご磼認じ利用ください。; This is not the published version. Please cite only the published version. 


\title{
Photoresponsive DNA Nanocapsule Having an Open/Close System for
}

\section{Capture and Release of Nanomaterials}

\author{
Tomohiro Takenaka,${ }^{[b]}$ Masayuki Endo, ${ }^{[a][c]}$ Yuki Suzuki, ${ }^{[b]}$ Yangyang Yang, ${ }^{[b]}$ Tomoko Emura, ${ }^{[b]}$ Kumi \\ Hidaka, ${ }^{[\mathrm{a}]}$ Takayuki Kato, ${ }^{[\mathrm{d}]}$ Tomoko Miyata, ${ }^{[\mathrm{d}]}$ Keiichi Namba, ${ }^{[\mathrm{d}]}$ and Hiroshi Sugiyama*[a][b][c]
}

\begin{abstract}
A photofunctionalized square bipyramidal DNA nanocapsule (NC) was designed and prepared. Photocontrollable open/close system and toehold system were introduced into the NC for the inclusion and release of a gold nanoparticle (AuNP) by photoirradiation and strand displacement. The reversible open and closed states were examined by gel electrophoresis and atomic force microscopy (AFM), and the open behavior was directly observed by high-speed AFM. The encapsulation of the DNAmodified AuNP into the NC was carried out via hybridization of a specific DNA (capture strand), and the release of the AuNP was examined by addition of toehold-containing complementary DNA (release strand). The release of the AuNP from the NC was successfully achieved by the opening of the NC and subsequent strand displacement
\end{abstract}

Nano-sized signal-responsive devices are attracting attention because of their potential applications for imaging, diagnosis, and therapeutics. ${ }^{[1,2]}$ The programmed DNA structure formation using the DNA origami method enables the construction of various predesigned DNA architectures. ${ }^{[3-5]}$ Because of the facility of DNA oligonucleotide functionalization, many researchers have reported the incorporation of functionalities into DNA nanostructures. ${ }^{[5-7]}$ Functional molecules and nanoparticles were incorporated into three-dimensional (3D) DNA nanostructures; ${ }^{[7,8]}$ however, many of these are static. Therefore, regulating configurational changes of $3 \mathrm{D}$ structures using a dynamic system is still a challenging issue, which can be applied for the expression of some biological activities. ${ }^{[9-11]}$

A sequential strand displacement using a toehold system was used for the operation of the DNA structural changes. ${ }^{[12]}$ We used the photoresponsive oligonucleotide (ODN) developed by Liang and Asanuma ${ }^{[13,14]}$ to assemble and disassemble DNA nanostructures by photoirradiation. ${ }^{[15-17]}$ In addition, reversible

[a] Prof. Dr. M. Endo, K. Hidaka, Prof. Dr. H. Sugiyama Institute for Integrated Cell-Material Sciences (WPI-iCeMS) Kyoto University

Yoshida-ushinomiyacho, Sakyo-ku, Kyoto 606-8501 (Japan) E-mail: endo@kuchem.kyoto-u.ac.jp; hs@kuchem.kyoto-u.ac.jp

[b] T. Takenaka, Dr. Y. Suzuki, Dr. Y. Yang, T. Emura, Prof. Dr. H. Sugiyama

Department of Chemistry, Graduate School of Science Kyoto University

Kitashirakawa-oiwakecho, Sakyo-ku, Kyoto 606-8502 (Japan)

[c] Prof. Dr. M. Endo, Prof. Dr. H. Sugiyama CREST

Japan Science and Technology Corporation (JST) Sanbancho, Chiyoda-ku, Tokyo 102-0075 (Japan)

[d] Dr. T. Kato, Dr. T. Miyata, Prof. Dr. K. Namba Graduate School of Frontier Biosciences Osaka Uniersity

1-3 Yamadaoka, Suita, Osaka 565-0871 (Japan)

Supporting information for this article is given via a link at the end of the document. dissociation and hybridization of photoresponsive ODNs by photoirradiation occurred in the DNA origami structure at the single-molecule level. ${ }^{[18]}$

In this work, a novel square bipyramidal DNA nanocapsule (NC) with a photoresponsive open/close system was designed and prepared. A pair of photoresponsive ODNs (PR-ODN 1 and PR-ODN 2) can hybridize in the trans-form of azobenzene and dissociate in the cis-form by photoirradiation at different wavelengths. ${ }^{[14]}$ The photoresponsive NC (PR-NC) can be opened by ultraviolet (UV) irradiation, and closed by subsequent visible light (Vis) irradiation, as illustrated in Figure 1a. In addition, we demonstrate the capture and release of a gold nanoparticle (AuNP) using a PR-NC with photoirradiation. We incorporated an AuNP attached to DNA into the opened PR-NC and included it by closing the PR-NC with Vis irradiation. Consequently, the PR-NC was opened by UV irradiation, and then the AuNP was released using strand displacement via a toehold system.
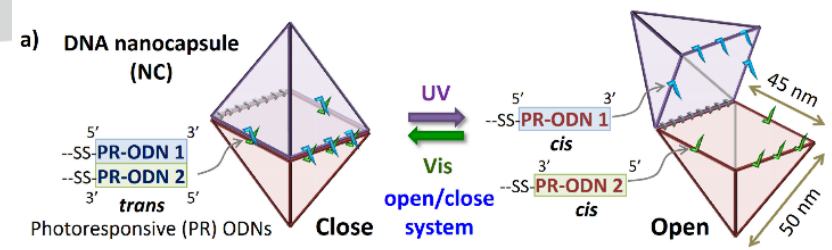

b)
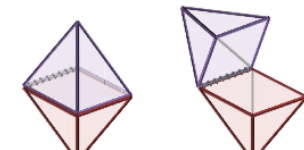

c)
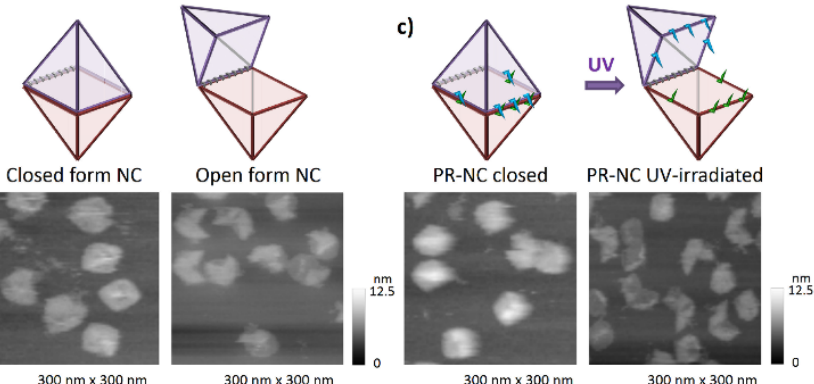

$300 \mathrm{~nm} \times 300 \mathrm{~nm}$

d)

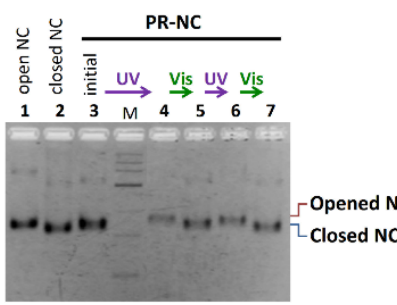

e)

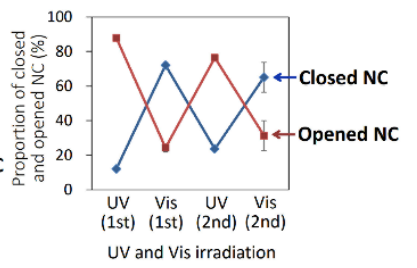

Figure 1. Photoresponsive DNA nanocapsule (PR-NC) with open/close system. (a) Schematic presentation of the PR-NC showing configuration changes between the open and closed states by UV and visible light (Vis) irradiation, respectively. (b) AFM images of the open and closed form NC. (c) AFM images of PR-NC in the initial state (closed) and after UV irradiation. (d) Agarose gel electrophoresis of open and closed NC with sequential UV/Vis irradiation. Lane M: DNA marker. (e) The yield of the closed and open PR-NCs by sequential UV/Vis irradiation. The proportions were obtained by counting the number of the closed and open states of NCs in the AFM images. 
We first prepared the DNA NC in both closed and open forms using the unmodified staple strands. The NCs were prepared by mixing $\mathrm{M} 13 \mathrm{mp} 18$ with five-equivalent staple strands in a buffer containing $20 \mathrm{mM}$ Tris buffer (pH 7.6), $10 \mathrm{mM} \mathrm{MgCl}_{2}$ and $1 \mathrm{mM}$ EDTA, and the mixture was annealed from $85^{\circ} \mathrm{C}$ to $65^{\circ} \mathrm{C}$ at a rate of $-1.0{ }^{\circ} \mathrm{C} / \mathrm{min}$, then from $65^{\circ} \mathrm{C}$ to $15^{\circ} \mathrm{C}$ at a rate of $-0.2^{\circ} \mathrm{C} / \mathrm{min}$. The assembled structure was imaged by AFM in the same buffer solution, and the formation of the structures was confirmed after removing the excess staple strands (Figures $1 \mathrm{~b}$ and S1). In the closed NC, a double-layered square was mainly observed, showing that the square bipyramidal structures were compressed on the mica surface. On the other hand, in the AFM images of the open NC, two kinds of open structures were observed; one was a single-layered fully opened structure with two connected squares, and the other was a double-layered half-opened structure that was attached by the side face on the mica surface (Figures $1 \mathrm{~b}$ and S2). These results showed that the closed and open NCs could be easily identified from the different appearances of the structures in the AFM images. The dimension of the NC was estimated from the AFM image of halfopened NCs (Figure S3). The bottom edge of the pyramid was $45.0 \pm 0.8 \mathrm{~nm}$, which was the expected length of $128 \mathrm{bp}$ dsDNA $(45 \mathrm{~nm})$. The side edge of the pyramid containing bundled 13 dsDNAs was $49.6 \pm 3.3 \mathrm{~nm}$, in which the interval of the bundled

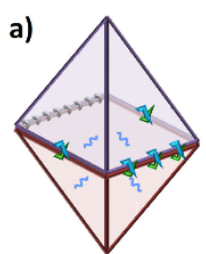

b)

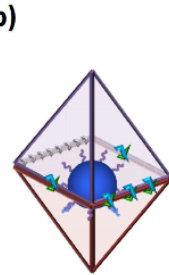

Closed PR-NC with AuNP
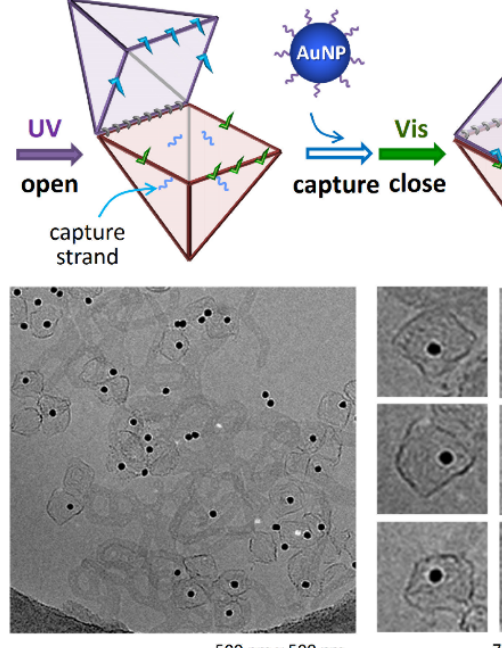

d)
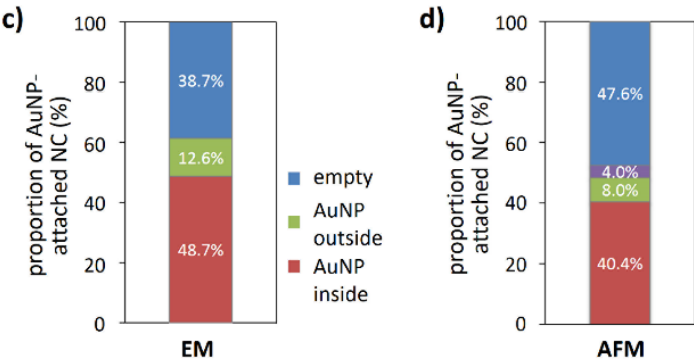

dsDNAs was $3.8 \mathrm{~nm}$

Figure 2. Capture of DNA-modified AuNP via hybridization of DNA strands inside the PR-NC. (a) The pre-UV-irradiated open PR-NC with four capture strands was treated with AuNP having complementary DNA strands to incorporate into the PR-NC. Then, the AuNP was encapsulated by closing using Vis irradiation. (b) Cryo electron microscope (EM) images of encapsulation of AuNP inside the PR-NC. Images on the right are individual AuNP-NCs. (c) Proportion of AuNP-attached PR-NCs obtained from the EM images $(N=119)$. (d) Proportion of AuNP-attached PR-NCs obtained from the AFM images $(N=118)$.

For incorporation of the PR-ODNs to the NC, the PR-ODNs were connected to the specific staple strands via disulfide bond (Table S1). ${ }^{[18]}$ The staple-PR-ODN strands were incorporated into the edges of the top and bottom pyramids of the NC by annealing using the same conditions above. To improve the efficient closing, we incorporate three PR-ODN pairs in the front edge of the NC and additional two pairs in the both side edges. In the AFM image of thePR-NC in the initial state, a doublelayered square was mainly observed similar to the closed NC, indicating that the PR-ODNs tightly closed the top and bottom pyramidal structures (Figures $1 \mathrm{c}$ and $\mathrm{S} 4$ ). Then, the closed PRNC was treated with UV irradiation for $5 \mathrm{~min}$, and the opened structures were observed in $88 \%$ including fully opened and half opened structures (Figures 1c and S5). These results indicated that the PR-ODNs control the open/closed state of the NC by photoirradiation. The reversible open/close behaviors of the PRNC were examined by gel electrophoresis (Figure 1d). Comparing with the mobility of the closed and open states of the NC using unmodified staples, the mobility of the PR-NC with UV and Vis irradiation was similar to the open and closed unmodified NCs, respectively. These results showed that the open and closed states of the PR-NC could be controlled by UV and Vis irradiation, respectively. The open/close system was also examined by counting the number of open and closed PRNCs in the AFM images (Figure 1e). The reversible open/close switching was observed using sequential UV and Vis irradiation. We also tried to observe opening of the PR-NC using highspeed AFM. ${ }^{[19-22]}$ The PR-NP remained closed during the AFM scanning, and then the closed PR-NC opened immediately after UV irradiation (Figure S6). The opening event of the PR-NP with UV irradiation can be directly visualized by high-speed AFM.

We next tried to introduce AuNP inside the PR-NC (Figure 2). AuNP has been used for demonstrating of its incorporation to the nanocages. ${ }^{[8,23-25]}$ To include AuNP inside, we introduced four single-stranded DNAs (capture strands) which were connected to the staple strands to the bottom pyramid of the PRNC cavity. The assembled NC was exposed to UV to open the structure. Then, two-equivalent of a $10 \mathrm{~nm}$ AuNP modified with the complementary strand was added to the opened PR-NC to introduce AuNP by hybridization. After incubation with AuNP under UV irradiation, the NC was closed by Vis irradiation to encapsulate the AuNP. In the gel electrophoresis image, the slower migrating band appeared compared with the empty PRNC (Figure S7). The sample was observed by cryo electron microscopy (EM) and AFM. In the EM images, we found the AuNP-attached PR-NCs and empty PR-NCs (Figures 2b and S8). Attachment of AuNP were observed inside and outside the NCs. The number of AuNP-attached PR-NCs was counted from the EM images. In the EM images, $49 \%$ of the NCs included AuNP in the NC. The attachment of AuNP outside the NCs should occur by hybridization to the closed PR-NC, and $13 \%$ of the NCs had AuNP outside. AuNPs attached outside the NCs were also observed in $12 \%$ of the samples. We also observed the PR-NCs with AuNPs attached by AFM. During the multiple 
AFM scanning, the AuNP-including NCs were opened, and the opened AuNP-attached NCs were counted (Figure S9). The yield of NCs with AuNP encapsulated was $\sim 40 \%$, and empty NCs were observed in $48 \%$ of the samples (Figure 2d). AuNPs attached outside the NCs were found in $8 \%$ of the samples, and those attached at unclear positions to the NCs were observed in $4 \%$ of the samples. From the results of both EM and AFM images, the AuNP encapsulated in the NC yielded over $40 \%$. We also examined the AuNP binding to the initial PR-NC (closed). AuNP was incubated with the PR-NC, and we found that $18 \%$ of the NC carried AuNP, in which $4 \%$ of AuNP bound outside the closed NC and $10 \%$ of AuNP bound to the halfopened NC (Figure S10). The capture strands can also trap
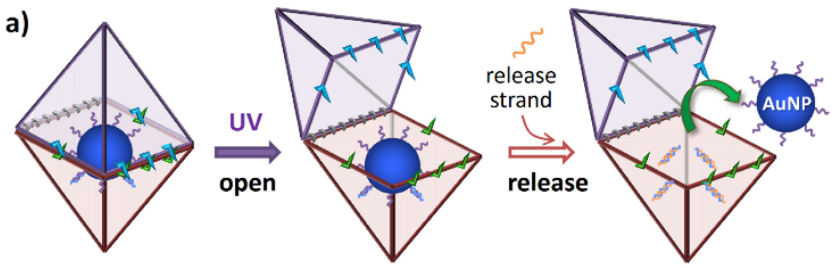

b)

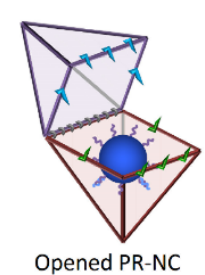
with AuNP
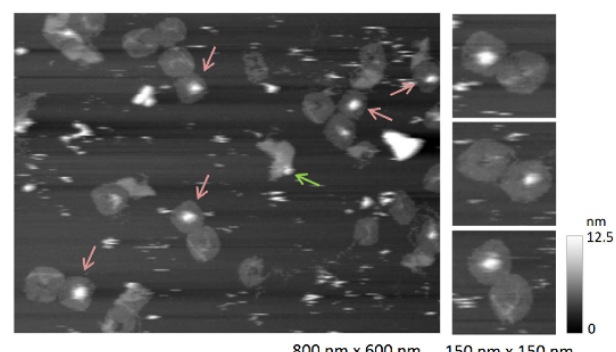

c)

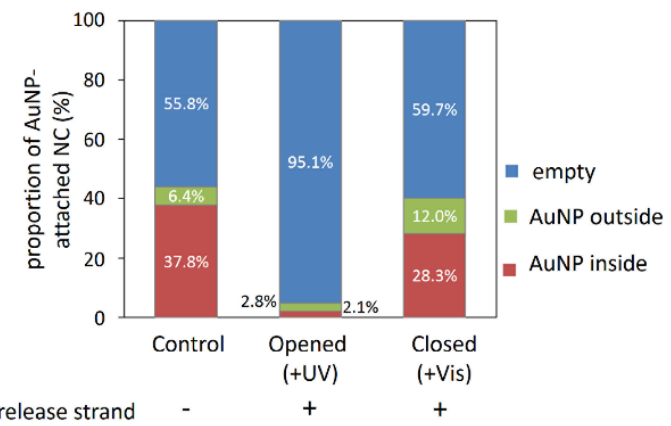

AuNP outside the NC, probably because the capture strands thread the gap space of the DNA origami. ${ }^{[26]}$ Also AuNP should bind to an opened NC that was a minor product of PR-NC, because the closing of the PR-NC was not perfect due to the incomplete trans-cis photoisomerization of the azobenzene molecule. ${ }^{[18]}$

Figure 3. Release of AuNP from the PR-NC by strand displacement. (a) For releasing AuNP, the closed PR-NC with encapsulated AuNP was opened by UV irradiation followed by adding a toehold-containing DNA strand. (b) AFM images of the AuNP-attached PR-NC after UV irradiation. Images on the right are individual opened AuNP-NCs. (c) The yield of AuNP after addition of the release strand. The release strand was incubated with the open (UVirradiated; $N=144$ ) and closed (Vis-irradiated; $N=184$ ) AuNP-attached PR-NCs. The yield of AuNP attachment after UV-irradiation is used as a control.

We then tried to release the AuNPs attached to the PR-NCs by opening and releasing using strand displacement (Figure 3a).
First, the closed PR-NCs were exposed to UV light to open, and the irradiated samples were observed by AFM. We found that AuNP was attached at the center of one of the square pyramids of the opened PR-NC (Figures $3 b$ and S11). The number of AuNPs attached to the center in the opened PR-NCs was counted $(N=204)$. Around $44 \%$ of the PR-NCs had AuNP, and $86 \%$ of the opened PR-NCs had AuNP at the center (Figure 3c). These results indicated that $38 \%$ of AuNP was included inside the NCs when AuNPs were incorporated into the opened PRNCs. We also observed the opening events of the AuNPencapsulated PR-NCs using high-speed AFM (Figure S12). The AuNP-encapsulated PR-NPs opened under UV irradiation. The AuNPs appeared at the center of the pyramid of the opened PRNP during AFM scanning, and remained attached inside the NC after the PR-NC opened.

Finally, the release of AuNPs was examined by strand displacement using toehold-containing DNA strands. In the previous reports, the release of AuNPs from the DNA nanostructures has been achieved by strand displacement. ${ }^{[23]}$ PR-NCs with AuNPs attached were closed by Vis irradiation, and then the release strand containing the toehold part was added to the sample after UV irradiation (Figure 3a). The number of PR-NPs in which AuNP remained attached was counted and compared with that before the addition of the release strand. The amount of AuNPs attached to the PR-NC dramatically decreased after UV irradiation and incubation with the release strand, and $\sim 90 \%$ of AuNPs were removed from the PR-NCs (Figures $3 \mathrm{c}$ and S13). Interestingly, when the closed PR-NC was incubated with the release strand, the release of AuNPs was suppressed (Figure 3c). These results indicated that the opened PR-NCs allowed hybridization of the release strand to detach AuNPs, whereas the closed PR-NCs prevent strand displacement by the release strand and the consequent release of AuNPs.

In conclusion, a square bipyramidal DNA NC was designed and constructed, and a photocontrollable open/close system was introduced into the NC. The reversible open/closed state of PR-NC was switched by Vis and UV irradiation. The inclusion of AuNPs into the PR-NC was also observed via hybridization of DNA strands between the NCs and AuNPs. In addition, the release of AuNPs was successfully controlled using photoirradiation and strand displacement. These nano-size PRNCs could be applied for an intelligent carrier for delivery of nanomaterials to cells similar to a virus capsid.

\section{Acknowledgements}

This work was supported by Core Research for Evolutional Science and Technology (CREST) of JST and JSPS KAKENHI (Grant Numbers 24310097, 24104002, 25253004, 26620133). Financial supports from The Mitsubishi Foundation and The Asahi Glass Foundation to ME are also acknowledged.

Keywords: DNA nanostructure - DNA origami • photocontrol • strand displacement $\bullet$ gold nanoparticle 
[1] H. Liang, X. Zhang, Y. Lv, L. Gong, R. Wang, X. Zhu, R. Yang, W. Tan, Acc. Chem. Res. 2014, 47, 1891-1901.

[2] J. A. Barreto, W.O'Malley, M. Kubeil, B. Graham, H. Stephan, L. Spiccia, Adv. Mater. 2011, 23, $\mathrm{H} 18-\mathrm{H} 40$.

[3] P. W. Rothemund, Nature, 2006, 440, 297-302.

[4] N. C. Seeman, Annu. Rev. Biochem. 2010, 79, 65-87.

[5] M. Endo, Y. Yang, H. Sugiyama, Biomater. Sci. 2013, 1, 347-360.

[6] A. Rajendran, M. Endo, H. Sugiyama, Angew. Chem. Int. Ed. 2012, 51, 874-890.

[7] J. Fu, M. Liu, Y. Liu, H. Yan, Acc. Chem. Res. 2012, 45, 1215-1226.

[8] Z. Zhao, E. L. Jacovetty, Y. Liu, H. Yan, Angew. Chem. Int. Ed. 2011, 50, 2041-2044.

[9] S. M. Douglas, I. Bachelet, G. M. Church, Science, 2012, 335, 831-834.

[10] M. Endo, R. Miyazaki, T. Emura, K. Hidaka, H. Sugiyama, J. Am. Chem. Soc. 2012, 134, 2852-2855.

[11] A. Kuzuya, Y. Sakai, T. Yamazaki, Y. Xu, M. Komiyama, Nat. Commun. 2011, 2, 449.

[12] J. Bath, A. J. Turberfield, Nat. Nanotechnol. 2007, 2, 275-284.

[13] H. Asanuma, X. Liang, H. Nishioka, D. Matsunaga, M. Liu, M. Komiyama, Nat. Protoc. 2007, 2, 203-212.

[14] X. Liang, T. Mochizuki, H. Asanuma, Small, 2009, 5, 1761-1768.

[15] F. Tanaka, T. Mochizuki, X. Liang, H. Asanuma, S. Tanaka, K. Suzuki, S. Kitamura, A. Nishikawa, K. Ui-Tei, M. Hagiya, Nano Lett. 2010, 10, 3560-3565.

[16] Y. Yang, M. Endo, K. Hidaka, H. Sugiyama, J. Am. Chem. Soc. 2012, 134, 20645-20653.

[17] Y. Suzuki, M. Endo, Y. Yang, H. Sugiyama, J. Am. Chem. Soc. 2014, 136, 1714-1717.

[18] M. Endo, Y. Yang, Y. Suzuki, K. Hidaka, H. Sugiyama, Angew. Chem. Int. Ed. 2012, 51, 10518-10522.

[19] T. Ando, N. Kodera, E. Takai, D. Maruyama, K. Saito, A. Toda, Proc. Natl. Acad. Sci. USA, 2001, 98, 12468-12472.

[20] A. Rajendran, M. Endo, H. Sugiyama, Chem. Rev. 2014, 114, 1493-1520.

[21] M. Endo, K. Hidaka, T. Kato, K. Namba, H. Sugiyama, J. Am. Chem. Soc. 2009, 131, 15570-15571.

[22] M. Endo, K. Hidaka, H. Sugiyama, Org. Biomol. Chem. 2011, 9, 20752077.

[23] Zhang, C.; Li, X.; Tian, C.; Yu, G.; Li, Y.; Jiang, W.; Mao, C. ACS Nano, 2014, 8, 1130-1135.

[24] D. Bhatia, S. Mehtab, R. Krishnan, S. S. Indi, A. Basu, Y. Krishnan, Angew. Chem. Int. Ed. 2009, 48, 4134 -4137.

[25] P. K. Lo, F. Altvater, H. F. Sleiman, J. Am. Chem. Soc. 2010, 132, $10212-$ 10214.

[26] N. Wu, D. M. Czajkowsky, J. Zhang, J. Qu, M. Ye, D. Zeng, X. Zhou, J. $\mathrm{Hu}$, Z. Shao, B. Li, C. Fan, J. Am. Chem. Soc. 2013, 135, 1217212175. 


\section{Entry for the Table of Contents}

\section{COMMUNICATION}

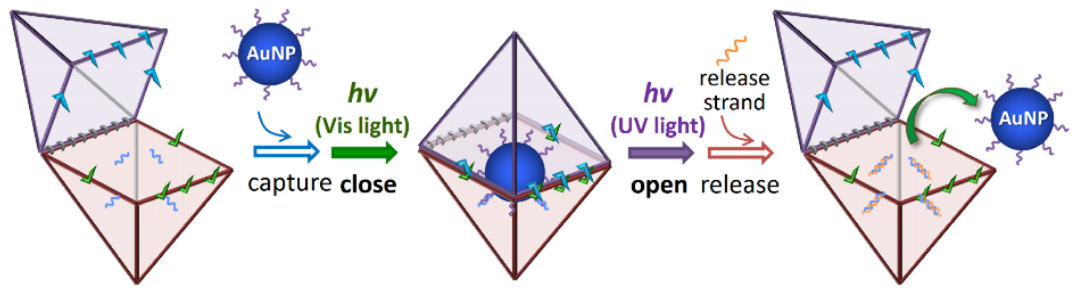

A square bipyramidal DNA nanocapsule (NC) was designed and prepared. Photocontrollable open/close system and toehold system were introduced into the $\mathrm{NC}$ for the inclusion and release of a gold nanoparticle (AuNP) by photoirradiation and strand displacement. The release of the AuNP from the NC was successfully achieved by the opening of the NC and subsequent strand displacement.
Tomohiro Takenaka, Masayuki Endo, * Yuki Suzuki, Yangyang Yang, Tomoko Emura, Kumi Hidaka, Takayuki Kato, Tomoko Miyata, Keiichi Namba, and Hiroshi Sugiyama*

Page No. - Page No.

Photoresponsive DNA Nanocapsule Having an Open/Close System for Capture and Release of Nanomaterials 